\title{
The Role of Calcium on the Active Site of Snake Venom Phospholipase A2: Molecular Dynamics Simulations
}

\author{
Akubugwo Emmanuel I. ${ }^{1}$, Okafor Irene N. ${ }^{2}$, Ezebuo Fortunatus C. ${ }^{2,}$, , Lukong Colin B. ${ }^{2}$, \\ Ifemeje Jonathan C. ${ }^{2}$, Nwaka Andrew C. ${ }^{2}$, Chilaka Ferdinand C. ${ }^{3}$ \\ ${ }^{1}$ Department of Biochemistry, Abia State University, Uturu, Nigeria \\ ${ }^{2}$ Department of Biochemistry, Faculty of Natural Sciences, Chukwuemeka Odumegwu Ojukwu University, Uli, Anambra State, Nigeria \\ ${ }^{3}$ Department of Biochemistry, Faculty of Biological Sciences, University of Nigeria, Nsukka, Enugu State, Nigeria
}

\section{Email address:}

fortunatus.ezebuo@unn.edu.ng (Ezebuo F. C.), ezebuofc@gmail.com (Ezebuo F. C.)

\section{To cite this article:}

Akubugwo Emmanuel I., Okafor Irene N., Ezebuo Fortunatus C., Lukong Colin B., Ifemeje Jonathan C., Nwaka Andrew C., Chilaka Ferdinand C. The Role of Calcium on the Active Site of Snake Venom Phospholipase A2: Molecular Dynamics Simulations. Computational Biology and Bioinformatics. Vol. 4, No. 1, 2016, pp. 10-14. doi: 10.11648/j.cbb.20160401.12

\begin{abstract}
Snake venoms are rich in phospholipase A2 (PLA2) and their hydrolysis of cell membrane phospholipids explains the role of the enzyme in venom toxicity. Calcium is known to plays important role at the active site of PLA2 during catalysis. In this study, molecular dynamics simulations of free PLA2 and calcium bound PLA2 were carried out using GROMACS 4.5.5 to evaluate the role of calcium in PLA2 catalysis. The results showed that calcium induced formation of helical structures between Arg62 - Lys66, Asn107 - Tyr111 and Asp114 - Cys119 in PLA2 which with time disappeared through the formation and opening of loops. Calcium induced atomistic movements and conformational changes in snake venom PLA2 which led to the formation of a widened cleft at the active site of calcium bound PLA2 when compared with free PLA2. This could lead to a better binding and accommodation of substrate, thus enhancing catalysis. This study confirms the role of calcium towards the action of PLA2 in snake venom toxicity and could provide useful information for the design of small molecules that can function as PLA2 inhibitors.
\end{abstract}

Keywords: Snake Venoms, Phospholipase A2, Calcium, Catalytic Site, Inhibitors of PLA2, Loop

\section{Introduction}

Phospholipases A2 (PLA2) (EC.3.1.1.4) catalyze the hydrolysis of the 2-acyl ester linkage of 1, 2-diacyl-3-snphosphoglycerides, with calcium $\left(\mathrm{Ca}^{2+}\right)$ requirement $[1,2]$ producing fatty acids and lysophospholipids [3]. Snake venoms are particularly rich in PLA2s [4] and PLA2s are able to induce several biological effects, such as pre-synaptic or post-synaptic neurotoxicity, cardiotoxicity, myotoxicity, platelet aggregation, oedema, haemolysis, anticoagulation, convulsion, and hypotension [5]. Their catalytic activity upon cell membranes of tissues suggests an important role of the enzyme in venom toxicity [6]. It has been reported elsewhere [3] that Asp49 is required for catalysis on artificial substrates. Also, studies have shown that snake venoms PLA2 need submicromolar concentrations of $\mathrm{Ca}$ to be catalytically active and also increase in $\mathrm{Ca}$ is needed for both binding and catalysis [7, 8]. A researcher [9] has described the calcium binding loop in myotoxicity of PLA2 and concluded that calcium binds to His48 of PLA2 located at the catalytic site of the enzyme activating it towards its toxic function. Also, His48 is involved in catalysis through orientation of its N-1 group to solvent [10]. Group II PLA2s display a binding site for $\mathrm{Ca}^{2+}$, which is highly conserved (X28CGXGG33) in addition to the highly conserved catalytic site (D42XCCXXHD49) previously reported [11]. The $\mathrm{Ca}^{2+}$ binding site is formed by the B-carboxyl group of Asp49 and the carbonyl groups of Tyr28, Gly30 and Gly32 [12]. The active site of snake venom PLA2 has been studied but the role of calcium on the dynamical properties of the catalytic site of the enzyme is limited. This study investigated the role of calcium on dynamical properties of snake venom phospholipase $\mathrm{A} 2$ by molecular dynamics simulations.

\section{Methodology}

\subsection{Molecular Dynamics Simulation}

GROMACS 4.5.5 was employed to carry out molecular 
dynamics simulations of free phospholipase A2 and its complex with calcium using Gromos53a6 force-field parameters [13]. The crystal structure coordinates of PLA2 obtained from RCSB Protein Databank (PDB ID: 3NJU) was considered for the simulation of free PLA2 and calcium bound PLA2 after editing with UCSF chimera 1.9 [14]. Both free and calcium bound structures of PLA2 was inserted into a simulation box with minimum $15 \AA$ distance between the box edge [15]. The boundaries were treated with periodic boundary condition involving the immersion of the simulation unit in periodic images of itself in the $\mathrm{x}, \mathrm{y}$ and $\mathrm{z}$ directions. The setup was subjected to 225 steps of in vacuo energy minimization using steepest descent algorithm. Then the systems were solvated using pre-equilibrated coordinates of the SPC explicit water model [16]. Sodium and chloride ions $\left(\mathrm{Na}^{+}\right.$and $\left.\mathrm{Cl}^{-}\right)$were added to neutralize the system and to model physiological salt concentration of $154 \mathrm{mM}$. The system was further minimized using 300 steps of steepest descent algorithm followed by 50 ps of position restrained dynamics where proteins were kept fixed by adding restraining forces, but water molecules were allowed to move. The P-LINCS [17] algorithm was used to constrain bond lengths, allowing the use of 2 fs time steps. Short-range non-bounded interactions were truncated at $12 \AA$ and employed the particle-mesh Ewald method [18, 19] in computing the long-range electrostatic interactions. Final production simulations were performed in the isothermal isobaric (NPT) ensemble at $300 \mathrm{~K}$, using v-rescale [20] as external bath with a coupling constant of $0.1 \mathrm{ps}$. Pressure was kept constant ( 1 bar) by using the time-constant for pressure coupling of $0.5 \mathrm{ps}$ and Parrinello-rahman barostat [20] for pressure coupling. Both free phospholipase A2 and its complex with calcium were subjected to 5000 ps $(5.0 \mathrm{~ns})$ molecular dynamics simulations and conformations generated during the simulations were stored every 2 ps.

\subsection{Data Analysis}

All the analyses were carried out using the available trajectory analysis tools of GROMACS packages. Visualization of molecular coordinates and rendering was achieved with VMD version 1.9.1 [21] and graphs were plotted with xmgrace on Linux platform.

\section{Results and Discussion}

To explore the role of calcium on the structure and function of PLA2, dynamic structural properties such as root mean square deviation (RMSD) and radius of gyration ( $\mathrm{Rg})$ of both calcium free and calcium bound PLA2 were obtained from molecular dynamics (MD) simulations analysis and presented in Figure 1.

The RMSD of atoms of the simulated protein over time is a reliable parameter to analyze the stability of the system. As evident from Figure 1, the first 0.2 ns of both simulations were considered to be the equilibration phase where slight structural re-organization takes place and properties were averaged over the last $5.0 \mathrm{~ns}$ of the simulations. RMSD and
Rg are two important parameters that provide quantitative descriptions of changes in the tertiary structure of the simulated protein. Similar RMSD were observed for free and calcium bound PLA2, which were $0.2332 \pm 0.0212 \mathrm{~nm}$ and $0.2389 \pm 0.02080 \mathrm{~nm}$ respectively. The calculated $\mathrm{Rg}$ value for free PLA2 was $1.4526 \pm 0.0139 \mathrm{~nm}$, while that of calcium bound PLA2 was $1.4469 \pm 0.0125 \mathrm{~nm}$. Essentially unchanged values of RMSD and Rg of free and ligand bound PLA2 signify no significant structural change of the venom protein PLA2 in the presence of calcium. Although there were no significant structural changes of PLA2 in the presence and absence of calcium when averaged over the last $5 \mathrm{~ns}$ of the simulations but deviations at $0.562,1.258$ and 4916 ns (Figure 1) were of interest to mention. Therefore, snapshots of atomistic details as well as key interactions occurring in free and calcium bound PLA2 at 0.562, 1.258 and $4.916 \mathrm{~ns}$ were obtained and presented in Figure 2. Between $0.4-1.0$ ns of the simulation, calcium bound PLA2 was more stable than free PLA2 whereas between $1.1-1.5$ ns and $4.8-5.0$ ns free PLA2 was more stable than calcium bound PLA2 (Figure 1A). Figure 1B showed that $\mathrm{Rg}$ of calcium bound PLA2 between $0.4-1.0$ ns was lower than that of free PLA2 whereas between $1.1-1.5$ and $4.8-5.0 \mathrm{~ns}$ free PLA2 showed lower radius of gyration.

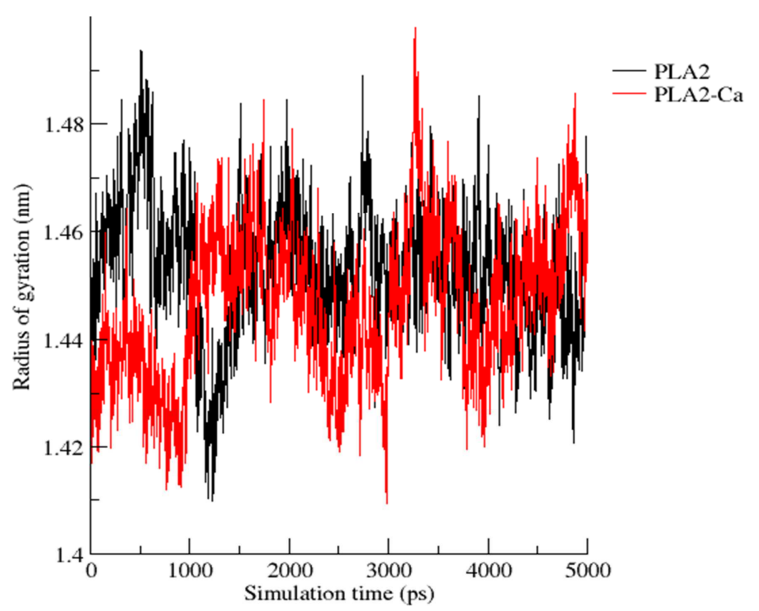

(A)

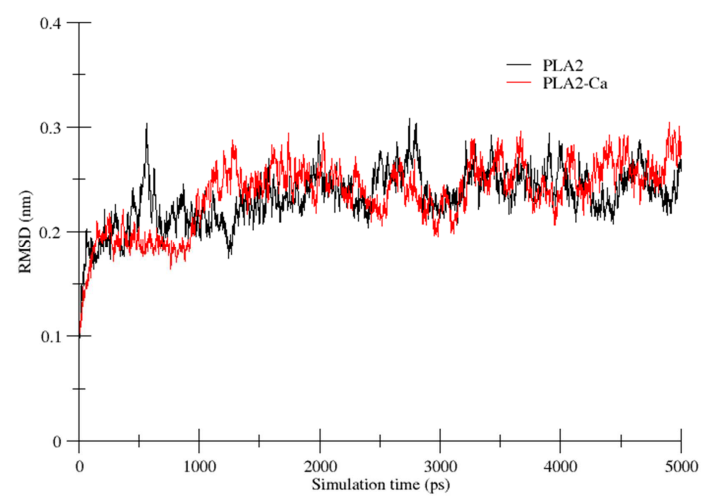

(B)

Figure 1. Variation of (A) RMSD and (B) Radius of gyration with simulation time for free PLA2 (black) and calcium bound PLA2 (red) obtained from molecular dynamics simulation. 
At $0.562 \mathrm{~ns}$, calcium bound PLA2 MD retained a helical structure (Helix 1) between Asp114 to Cys119 when compared with PLA2 structure at $0.0 \mathrm{~ns}$ which was not observed in the free PLA2 MD (Figure 2A). Also at $0.562 \mathrm{~ns}$, calcium bound PLA2 MD showed an additional helical structure (Helix 2) between Arg62 to Lys66 (Figure 2A). It is important to note that at $0.562 \mathrm{~ns} \mathrm{MD}$, the loops (Loop 1 and 2) of calcium free PLA2 deviated more than those of calcium bound PLA2 when compared with the loops of PLA2 at 0.0 ns (Figure 2). At 1.258 and $4.916 \mathrm{~ns}$, the helical structure (Helix 1) in calcium bound PLA2 disappeared and there was the appearance of a third helical structure (Helix 3) between Asn107 to Tyr111 which was not retained at $4.916 \mathrm{~ns}$ in calcium free PLA2 (Figure $2 \mathrm{~B}$ and C). Again at 1.258 and $4.916 \mathrm{~ns}$, the loops (Loop 1 and 2) of calcium bound PLA2 deviated more than that of calcium free PLA2 when compared with the loops of PLA2 at 0.0 ns (Figure $2 \mathrm{~B}$ and C). These results indicate that calcium initially induces formation of helical structures (between Arg62 to Lys66 and Asn107 to Tyr111) in snake venom PLA2 which starts to disappear with the formation and opening of loops (Figure 2). The observed relatively opened loop in calcium bound PLA2 indicate that calcium plays a crucial role in PLA2 loop flexibility and function. To have an idea of the regions of free and calcium bound PLA2 that are more flexible, the RMSF of free and calcium bound PLA2 were calculated and presented in Figure 3.
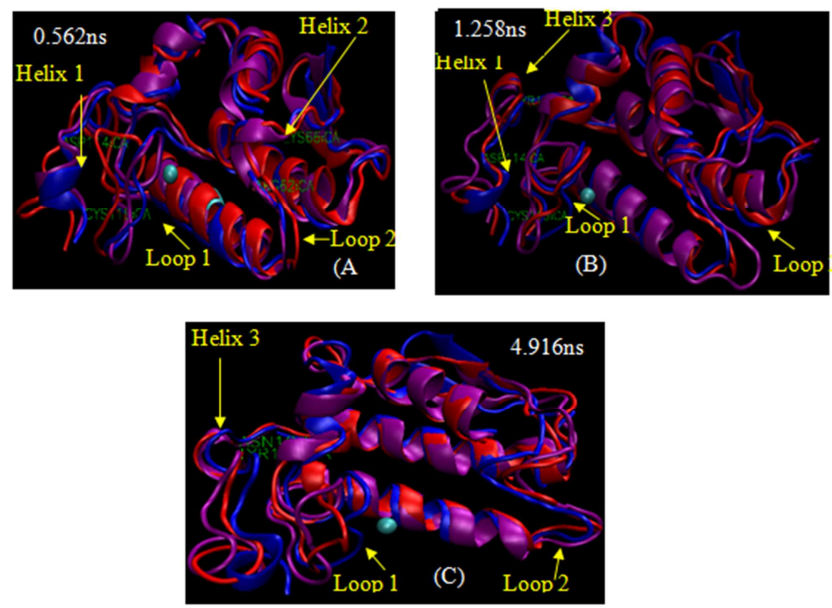

Figure 2. Snapshots of PLA2 obtained from molecular dynamics simulation at (A) $0.562 \mathrm{~ns}$, (B) $1.258 \mathrm{~ns}$ and (C) $4.916 \mathrm{~ns}$. Blue represent PLA2 at 0.0 $n s$, red is free PLA2 while purple is calcium bound PLA2. Loops and helical structures are coloured yellow. Calcium is represented as light green sphere.

From the RMSF results (Figure 3), calcium free PLA2 from snake venom showed more flexibility between amino acid residues of Val13 - Ser15, Asp21 - Phe22, and Cys79 - Lys80 while that of calcium bound PLA2 showed more flexibility between residue ids of Cys29 - Gly30 and Asn57 - Ser59 (Figure 3). This suggests that one of the roles of calcium in PLA2 is to increase flexibility of some essential amino acid residues. Another important aspect observed from the MD simulation was the distance between calcium and some amino acid residues at the catalytic site and most flexible regions of PLA2. The minimum distance between calcium and amino acids at the peaks of the flexible regions including some amino acids (His48, Asp49) at the catalytic site were calculated and the results presented in Figure 4.

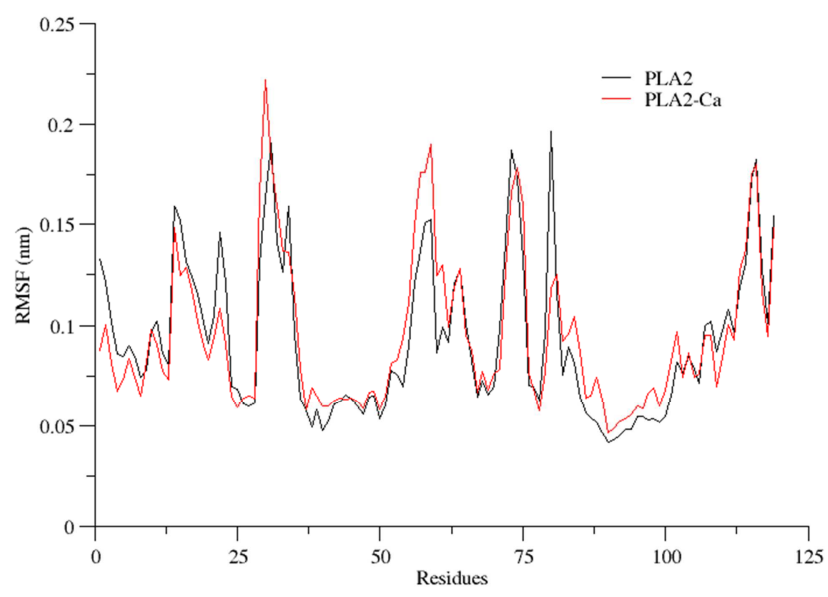

Figure 3. Variation of RMSF with simulation time for free PLA2 (black) and calcium bound PLA2 (red) obtained from molecular dynamics simulation.

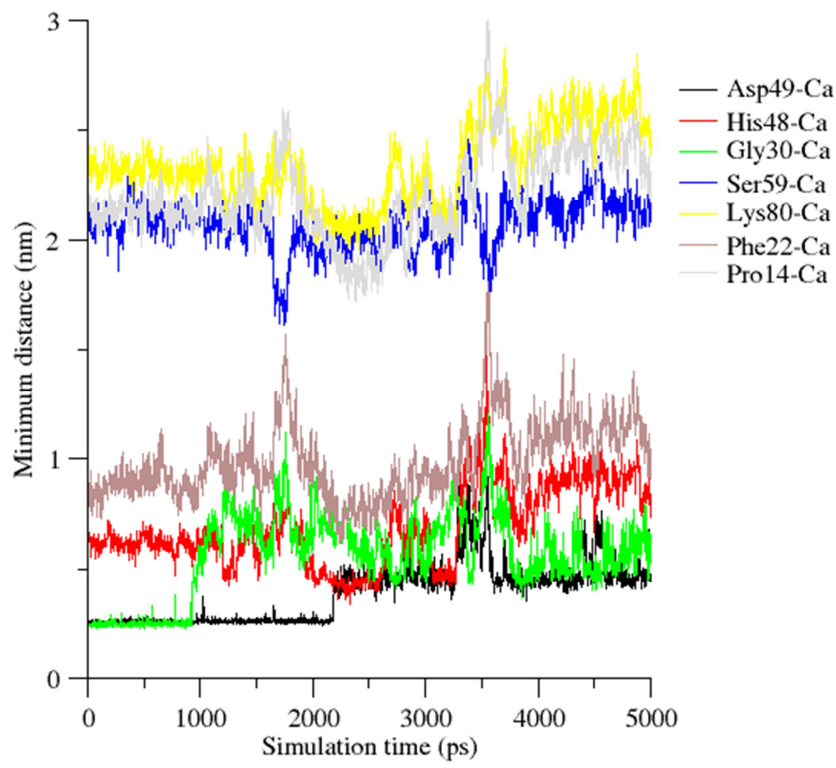

Figure 4. Minimum distance between calcium and some amino acids (Pro14, Phe22, Gly30, His48, Asp49, Ser59 and Lys80) during molecular dynamics simulation.

The minimum distances between calcium and some residues (Pro14, Phe22, Gly30, His48, Asp49, Ser59 and Lys80) were calculated during MD simulation and the results are shown in Figure 4. The results (Figure 4) showed that Asp49 maintained average distance of $0.2601 \pm 0.0092 \mathrm{~nm}$ from calcium during the first $2.186 \mathrm{~ns}$ of the simulation and $0.4906 \pm 0.0903 \mathrm{~nm}$ from 2.186 to $5.0 \mathrm{~ns}$ of the simulation. During the first $0.930 \mathrm{~ns}$ of the simulation, Gly30 maintained an average minimum distance of $0.2501 \pm 0.016 \mathrm{~nm}$ from calcium which increased to $0.6344 \pm 0.1322 \mathrm{~nm}$ for the last $5.0 \mathrm{~ns}$. It is also important to note that His48 had an average distance of $0.5787 \pm 0.1001 \mathrm{~nm}$ from calcium during the first $3.286 \mathrm{~ns}$ of the simulation which increased to $0.8918 \pm$ 
$0.1095 \mathrm{~nm}$ for the last $5.0 \mathrm{~ns}$. During the dynamics, the increase in the distance between calcium and the amino acids at the catalytic site and most flexible regions of PLA2 suggests that calcium stimulates the movement of amino acids at the catalytic site and most flexible regions of PLA2 to create a widened cleft to accommodate the substrate/ligand. Figure 5 depicts the extent of calcium on the widening of the active site of PLA2. This could explain the observations made elsewhere $[7,8]$ that snake venom PLA2 need submicromolar concentrations of $\mathrm{Ca}$ to be catalytically active and that increase in $\mathrm{Ca}$ is needed for both binding and catalysis. Also, it was observed elsewhere [22] that snake venom PLA2 activity was enhanced by Calcium and inhibited by Magnesium, Cobalt and Copper. They suggested that this could be as a result of probable modification of enzyme protein conformation after binding differently from the pattern calcium does, and hence causing a decrease in the enzyme action.
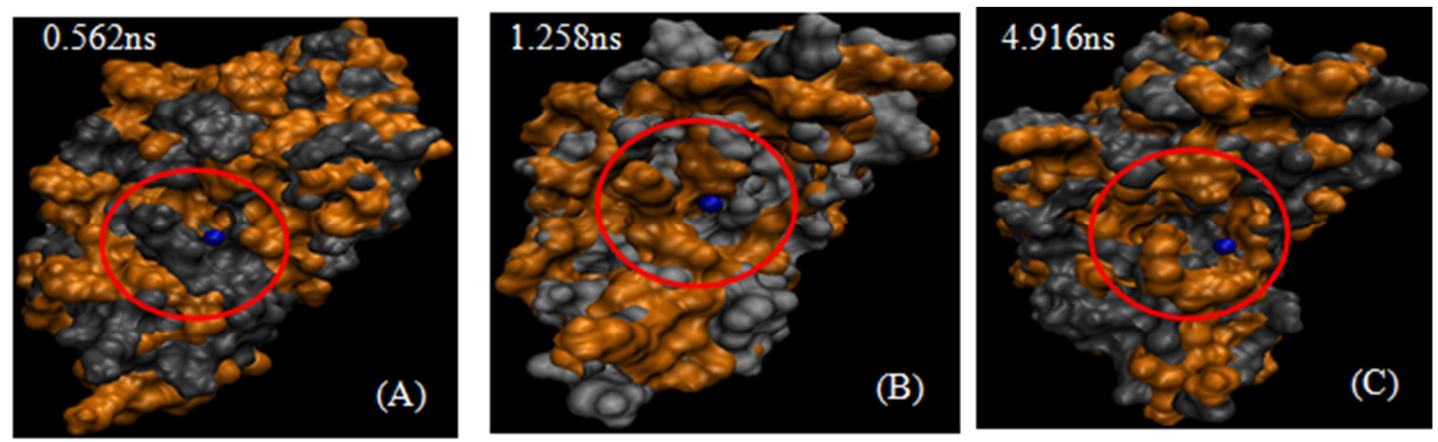

Figure 5. Snapshots of active site of PLA2 obtained from molecular dynamics simulation at (A) $0.562 \mathrm{~ns}$, (B) $1.258 \mathrm{~ns}$ and (C) $4.916 \mathrm{~ns}$. Orange is free PLA2 while gray is calcium bound PLA2. Calciumis represented as blue sphere while the region of the active site is represented as red circle.

Wider cleft were observed in calcium bound PLA2 than free PLA2 at 1.2582 and 4.916 (Figure $5 \mathrm{~B}$ and $\mathrm{C}$ ). The widening of the cleft was not observed at $0.562 \mathrm{~ns}$ of the simulation probably due to the initial formation of a helical structure (Helix 1) in calcium bound PLA2 but not in free PLA2 (Figure 2). The widening of the cleft could likely lead to better accommodation/binding of substrate, hence efficient catalysis.

\section{Conclusion}

Considering the results of this study, calcium induces atomistic movements and conformational changes in snake venom PLA2 which leads to formation of wider cleft at the active site of calcium bound PLA2 when compared with free PLA2. The results of this work have shed more light into the mechanism of action of calcium in snake venom toxicity and could be helpful to design small molecules which could function as novel inhibitors of PLA2 as none is available as drug in the market [23]. Such molecules would likely act by inhibiting the formation of loops or its flexibility and/or stabilization of the generated helical structures which would prevent the widening of the cleft at the active site of PLA2. Any molecule with such a mechanism of action would be a potent inhibitor of PLA2.

\section{References}

[1] Ohno, M., Ménez, R., Mgawa T., Manse, J. M., Shimohigashi Y., Fromen C., Ducancel, F., Zinn-justin S., Le du M. H., Boulain, J. C., Tamiya, T., Ménez, A. (1998) Molecular evolution of snake toxins: Is the functional diversity of snake toxins associated with a mechanism of accelerated evolution? In: MOLDAVE K. Ed. Progress in nucleic acid research and molecular biology. San Diego: Academic Press, 307-64.
[2] Slotboom, A. J., Verheij, H. M., De Hass, G. H. (1982) On the mechanism of phospholipasesA2. In: Hawthorne J. N., Ansell GB. Eds. Phospholipids, new biochemistry. Amsterdam: Elsevier Biomedical Press/ North Holland Press, 359-434.

[3] Yu, B. Z., Berg, O. G., Jain, M. K. (1993) The divalent cation is obligatory for the binding of ligands to the catalytic site of secreted PLA2. Biochemistry, 32, 6484-6492.

[4] Ramírez-Avila, J., Quevedo, B. E., López, E., Renjifo, J. M. (2004) Purification And Partial Characterization Of Phospholipases A2 From Bothropsasper (BARBA AMARILLA) Snake Venom From Chiriguaná (Cesar, Colombia). J. Venom. Anim. Toxins incl. Trop. Dis., 2004, 10, 3 , p. 243.

[5] Soares, A. M., Rodrigues, V. M., Homsi-Brandeburgo M. I., Tayoma, M. H., Lombardi, F. R., Arni, R. K., Giglio, J. R. (1998) A rapid procedure for the isolation of the Lys-49 myotoxin II from Bothrop smoojeni (Caissaca) venom: biochemical characterization, crystallization, myotoxic and edematogenic activity. Toxicon 36, 503-517.

[6] Ownby, C. L., Selistre-de-Araújo, H. S., White, S. P., Fletcher, J. E. (1999) Lysine 49 phospholipase A2 proteins. Toxicon, 37, 411-445.

[7] Shashidharamurthy, R., Kemparaju, K. (2006). A neurotoxic phospholipase A2 variant: Isolation and characterization from eastern regional Indian cobra (Najanaja). Toxicon. 47: 727733.

[8] Higuchi, D.A., Barbosa, C.M.V., Bincoletto, C., Chagas, J.R, Magalhaes A, Richardson M, Sanchez E.F., Pesquero J.B., Araujo, R.C, Pesquero, J.L. (2007). Purification and partial characterization of two phospholipases A2 from Bothrop sleucurus (white tailed-jararaca) snake venom. Biochemie. 89: 319-328.

[9] Yang, C. C (1994) Structure-function relationship of PLA2 from snake venoms. J. Toxicol. Toxin Rev. 13: 125-127. 
[10] Fukagawa, T., Nose, T., Shimohigashi, Y., Ogawa, T., Oda, N., Nakashima, K., Chang, C. C., Ohno, M. (1993) Purification, sequencing and characterization of single amino acidsubstituted phospholipaseA2 isozymes from Trimeresurusgramineus (green habu snake) venom. Toxicon, 31, 957-967.

[11] Murakami, M.; Kudo, I. (2002) Phospholipase A2. J. Biochem. 131, 285-292.

[12] Fleer, E. A., Verheij, H. M., De Haas, G. H. (1981) Modification of carboxylate groups in bovine pancreatic phospholipase A2. Identification of aspartate-49 as $\mathrm{Ca}^{2+}$ binding ligand. Eur. J. Biochem., 113: 283-288.

[13] Oostenbrink, C., Villa, A., Mark, A. E., Van Gunsteren, W. F. (2004) A biomolecular force fieldbased on the free enthalpy of hydration and solvation: The GROMOS force-field parametersets 53A5 and 53A6. Journal of Computational Chemistry 25(13): 1656-1676.

[14] Pettersen, E. F., Goddard, T. D., Huang, C. C., Couch, G. S., Greenblatt, D. M., Meng, E. C. and Ferrin, T. E. (2004) UCSF Chimera: A visualization system for exploratory research and analysis. J Comput Chem.; 25(13): 1605-12.

[15] Weber, W., Hunenbeger, P. H and Mc Cammon, J. A (2000) Molecular dynamics simulations of a polyalanineoctapeptide under Ewald boundary conditions: Influence of artificial periodicity on peptide conformation, J. Phys. Chem., B 104: $3575-3668$.

[16] Berendsen, H. J. C., Postma, J. P. M., van Gunsteren, W. F.,
Hermans, J. (1981) Interaction modelsfor water in relation to protein hydration. In: Intermolecular Forces. Pullman, B. ed.. D. Reidel Publishing Company Dordrecht 331-342.

[17] Hess, B. (2007) P-LINCS: A parallel linear constraint solver for molecular simulation. J. Chem. Theory Comp. 4: 116-122.

[18] Darden, T., York, D., Pedersen, L. (1993) Particle mesh Ewald: An N_log(N) method for Ewald sums in large systems. J. Chem. Phys. 98: 10089-10092.

[19] Essmann, U., Perera, L., Berkowitz, M. L., Darden, T., Lee, H., Pedersen, L. G. A (1995) smooth Particle Mesh Ewald potential. J. Chem. Phys. 103: 8577-8592.

[20] Bussi, G., Donadio, D., Parrinello, M. (2007) Canonical sampling through velocity rescaling. J. Chem. Phys. 126: 014101 .

[21] Humphery, W., Dalke, A. and Schulten K., (1996) VMD Visual Molecular Dynamics, J. Molec. Greaphics, 14: 33 - 38.

[22] Sallau, A. B., Ibrahim, M. A., Salihu, A. and Patrick, F. U. (2008) Characterization of phospholipase A2 (PLA2) from Echisocellatus venom African Journal of Biochemistry Research 2 (4), pp. 098-101.

[23] Yarla, NS., Satyakumar, K., Srinivasu, D., Kaladhar DSVGK, Aliev G, Dharmapuri, G., Putta, G. R. S., Jagarlapoodi, S., Bheeram, V., Sadus, S. P and Duddukuri G. R (2015) Phospholipase A2: A Potential Therapeutic Target in Inflammation and Cancer (In silico, In vitro, In vivo and Clinical Approach). J Cancer Sci Ther 7: 249-252. doi: 10.4172/1948-5956.1000357. 